\title{
Motivational interviewing versus feedback only in emergency care for young adult problem drinking
}

\author{
Peter M. Monti',2, Nancy P. Barnett ${ }^{2}$, Suzanne M. Colby ${ }^{2}$, Chad J. Gwaltney², Anthony Spirito², \\ Damaris J. Rohsenow ${ }^{1,2} \&$ Robert Woolard ${ }^{3}$ \\ Providence VA Medical Center, RI, USA,' Brown University Center for Alcohol and Addiction Studies, Providence, RI, USA² and Brown University Medical School, \\ Rhode Island Hospital, RI, USA ${ }^{3}$
}

\section{ABSTRACT}

Aim To establish the efficacy of a brief motivational intervention compared to feedback only when delivered in an emergency department for reducing alcohol use and problems among young adults. Design Two-group randomized controlled trial with follow-up assessments at 6 and 12 months. Setting Level I Trauma Center. Participants A total of 198 18-24-year-old patients who were either alcohol positive upon hospital admission or met screening criteria for alcohol problems. Intervention Participants were assigned randomly to receive a one-session motivational intervention (MI) that included personalized feedback, or the personalized feedback report only (FO). All participants received additional telephone contact 1 month and 3 months after baseline. Measurements Demographic information, alcohol use, alcohol problems and treatment seeking. Findings Six months after the intervention MI participants drank on fewer days, had fewer heavy drinking days and drank fewer drinks per week in the past month than did FO patients. These effects were maintained at 12 months. Clinical significance evaluation indicated that twice as many MI participants as FO participants reliably reduced their volume of alcohol consumption from baseline to 12 months. Reductions in alcohol-related injuries and moving violations, and increases in alcohol treatment-seeking were observed across both conditions at both follow-ups with no differences between conditions. Conclusions This study provides new data supporting the potential of the motivational intervention tested to reduce alcohol consumption among high-risk youth.

Keywords Alcohol, brief intervention, emergency room, motivational interviewing, young adults.

\section{INTRODUCTION}

The highest rates of alcohol consumption and problem drinking in the United States are among 18-25-year-olds, with approximately $41 \%$ of young adults engaging in heavy episodic drinking (five or more drinks on one occasion) in the past month [1]. Young adults have the highest rate of alcohol-related traffic deaths, and in the past 3 years unintentional alcohol-related deaths have increased significantly in 18-24-year-olds [2]. Despite their high level of risk, adolescents and young adults are less likely than older adults to perceive a need and less likely to present for treatment for alcohol and substance abuse [3].

As many as $47 \%$ of injured trauma patients have positive blood alcohol levels and approximately $40 \%$ of trauma patients with negative blood alcohol levels meet criteria for current alcohol dependence [4,5]. Urgent care settings provide an opportunity to reach young adults who might benefit from intervention, and brief interventions conducted in these settings have proved useful with adults in reducing both alcohol consumption and alcohol-related injuries when compared to controls $[6,7]$. Brief interventions for alcohol are recommended for medical settings [8] including emergency departments (ED; [9]), and are now mandated in trauma centers [10].

Brief intervention studies conducted in medical settings have typically followed guidelines of motivational interviewing (MI; [11]), which is a client-centered, directive method for enhancing intrinsic motivation to change by exploring and resolving ambivalence. MI is guided by 
four principles: expressing empathy, developing discrepancy, rolling with resistance and supporting self-efficacy. MI has proved effective in reducing problem drinking among adult drinkers [12,13] and college students (see [14] for review), and with adults, young adults and highrisk adolescents in the ED [15-17]. MI is particularly well suited for use in an ED in that it is used easily with patients at all levels of readiness to change [11]. Relative to no-treatment control groups the evidence in favor of using MI in the ED is strong.

Personalized feedback about a patient or client's alcohol use and alcohol-related risks is almost always included in brief interventions and MI sessions. Personalized feedback typically includes gender- and age-based normative information about alcohol use rates and specific information about personal risk levels [18,19]. Personal feedback per se does not require the presence of a counselor and its delivery takes considerably less time than a full MI. As a very brief but personalized and educational approach it is an ecologically valid alternative to in-person counseling, and in studies of college students there is support for its efficacy as a stand-alone intervention [20-22]. Given the personnel required to deliver MIs as well as the additional time required, above and beyond the time associated with personalized feedback, the question arises as to whether personalized feedback only would have the same impact as a full MI. This question is especially pertinent for working in an ED where time and clinical personnel are most valuable. Because this question has not been addressed in an ED setting, the present study was designed to do so.

The objective of this study was to examine the efficacy of a brief MI that included personalized feedback compared to a feedback only (FO) condition when delivered in an ED setting with a sample of young adult patients with drinking problems. We selected an active comparison condition without controlling for contact time, as MI has already been shown to be superior to a no-treatment group, and we wished to test it in a real-world setting against an intervention with proven efficacy in a similarage population. We provided booster sessions 1 and 3 months after the intervention for both conditions, as additional contact following treatment is recommended by clinical guidelines [23,24]. Given the additional counseling that the MI provides, we hypothesized that patients who received MI would show lower levels of consumption and alcohol-related problems at 6- and 12-month follow-up than those receiving FO.

\section{METHODS}

Patients between the ages of 18-24 years at a level 1 trauma center in Rhode Island were invited to participate in the study between January 2000 and June 2003 if they were treated in the ED and: (a) had a blood alcohol concentration (BAC) greater than $0.01 \%$ according to a biochemical test, (b) reported drinking alcohol in the 6 hours prior to the event that caused their visit or (c) scored 8 or higher on the Alcohol Use Disorders Identification Test (AUDIT; [25]). Excluded were patients who did not speak English, had a self-inflicted injury or were in police custody. After establishing eligibility, research counselors conducted a mini-mental status examination which included being able to remember and explain important components of the study. Counselors used the results of the mini-mental examination and clinical judgement to establish readiness for consent and assessment. All procedures were approved by the university and hospital Institutional Review Boards and all participants gave written informed consent. Counselors administered baseline assessments using a laptop computer (3045 minutes), after which patients were assigned randomly to a treatment condition (by the project coordinator using a random numbers table).

\section{Counselor training and supervision}

Counselors were nine bachelor's and master's level clinicians with previous experience. Counselor expertise level was intended to be similar to (non-research) clinicians who might conduct these interventions in medical settings. Counselors received approximately 30 hours of training in MI, which included readings, exercises, viewing demonstrations and extensive observed roleplaying of MI techniques and the specific project protocol. Weekly supervision in MI was conducted by the second author. Patient reports of therapist behavior (see below) were also reviewed to ensure standardization.

\section{Follow-up assessments}

Follow-up assessments were conducted 6 and 12 months after baseline by research assistants blind to intervention condition. Participants received a $\$ 25$ gift certificate for participating at baseline, $\$ 15$ cash for the 1-month booster, $\$ 30$ for the 3-month booster, $\$ 40$ each for 6- and 12-month follow-ups, and a $\$ 30$ bonus if all follow-ups were completed within 1 month of their due dates.

\section{Measures}

The time-line follow-back (TLFB; [26]) method was used to measure alcohol use for the 30 days prior to the ED visit, and prior to the 6-month and 12-month follow-up appointments. Number of days drinking, number of heavy drinking days ( $\geq$ five drinks for men, $\geq$ four for women), and average drinks per week were calculated. Alcohol-related consequences were measured using the Rutgers Alcohol Problem Index (RAPI; 27), which had a 
Table 1 Patient reports of motivational interview and feedback only sessions.

\begin{tabular}{|c|c|c|c|c|}
\hline Measure & $\begin{array}{l}M I(n=87) \\
M(S D)\end{array}$ & $\begin{array}{l}F O(n=91) \\
M(S D)\end{array}$ & $d f$ & $t$ \\
\hline The counselor was concerned about me & $3.69(0.49)$ & $3.42(0.70)$ & 176 & $3.01^{* *}$ \\
\hline The counselor helped me believe that I can change my drinking if I want to & $3.52(0.71)$ & $3.12(0.95)$ & 176 & $3.15^{* *}$ \\
\hline $\begin{array}{l}\text { The counselor made me feel that it is up to me to make decisions about my } \\
\text { drinking and what I do when I drink }\end{array}$ & $3.80(0.53)$ & $3.14(0.99)$ & 175 & $5.55^{* * *}$ \\
\hline $\begin{array}{l}\text { The counselor gave me some helpful suggestions about drinking or things } \\
\text { that happen when I drink }{ }^{\mathrm{a}}\end{array}$ & $3.66(0.65)$ & $2.64(1.13)$ & 141 & $6.64^{* * * *}$ \\
\hline The counselor was easy to talk to & $3.92(0.27)$ & $3.92(0.27)$ & 176 & -0.09 \\
\hline The counselor gave me the chance to ask questions ${ }^{\mathrm{a}}$ & $3.83(0.45)$ & $3.75(0.50)$ & 140 & 1.06 \\
\hline How satisfied were you with the session $?^{\mathrm{a}}$ & $4.65(0.69)$ & $4.58(0.60)$ & 141 & -0.18 \\
\hline
\end{tabular}

The first item was answered on a five-point scale from 1, 'not at all satisfied', to 5 , 'very satisfied'. The other items were answered on a four-point scale from 1 , 'strongly disagree', to 4 , 'strongly agree'. 'This item was added to the measure after the start of the trial, which resulted in a lower $n$. ** $P<0.01$; *** $P<0.001$.

3-year time-frame at baseline and a 6-month window at each follow-up (Cronbach's alpha $=0.90$ ). Other measures included a count of alcohol-related injuries (Adolescent Injury Checklist; [28]), frequency of driving within an hour of drinking one or more drinks [29] and five items measuring counseling or treatment for alcohol problems. For the injury, driving and treatment measures, information collected at baseline reflected the past year; 6- and 12-month follow-ups reflected the past 6 months and were combined to reflect the year of follow-up. Driving records from participants' State Department of Motor Vehicles were reviewed, from which driving violations, including alcohol-related violations, for the 1 year before and after baseline were extracted.

Patient perception of the interventions was measured by seven items reflecting session process and content (Cronbach's alpha $=0.70$; Table 1$)$. Patients who completed follow-up interviews provided the name of an informant who was contacted to provide a collateral report of the patient's alcohol use (number of heavy drinking days) and alcohol consequences using a modified RAPI.

\section{MI condition}

The MI (30-45 minutes) was delivered in the ED and incorporated open-ended exploration, personalized feedback, support for self-efficacy and discussion about patients' alcohol use and associated risky behaviors [30]. Session components included establishing rapport, assessing motivation for change, enhancing motivation and establishing goals for change. A worksheet was used to outline the patient's reasons for change and barriers to change. A graphic personalized feedback report was derived from the baseline assessments and included normative information about consumption, summaries of the patient's alcohol-related consequences and indicators of risk, and was discussed in the MI session to enhance motivation. If the patient was interested in discussing change, a goal worksheet containing reduction and cessation strategies was used to facilitate this process. Patients received copies of the worksheets and feedback report, and handouts about alcohol risks and local treatment facilities.

Telephone booster sessions were conducted 1 and 3 months after baseline. The 1-month booster (20 minutes) started with an assessment (30-day TLFB) of the patient's past-month drinking. The counselor then reviewed the patient's goals from the first session and inquired about progress. Goals were adjusted or new goals set. For the 3-month booster (25-30 minutes), patients completed a short assessment battery of alcohol consumption and problem measures described above. A new feedback sheet about the patient's recent behavior relative to their baseline report was generated and reviewed, and a copy mailed to the patient. Progress toward goals since the first booster was reviewed and new goals were generated and discussed.

\section{FO condition}

Patients in FO received the same baseline assessment and computer-generated personalized feedback report as those in MI. Counselors introduced the report as providing information about how much the patients drink and what happens when they drink, and how they compare to others their age. Conversation with the counselor was minimal, but any questions or concerns were addressed appropriately. Contact lasted 1-3 minutes. Patients received the same handouts as in MI. One month after baseline patients in the FO condition received a telephone call from the counselor, which consisted only of the assessment described in the MI section above (5-10 minutes). At 3 months FO patients completed the 
Table 2 Blood alcohol concentration (BAC) and AUDIT total score by trial entry status.

\begin{tabular}{lllr}
\hline Measure & $B A C+(n=111)$ & $\begin{array}{l}\text { Self-report }(n=40) \\
M(S D)\end{array}$ & $\begin{array}{l}\text { AUDIT (n=47) } \\
M(S D)\end{array}$ \\
\hline BAC & $M(S D)$ & $0.033(0.049)$ & $0.000(0.000)$ \\
AUDIT Total & $0.132(0.077)$ & $8.5(6.8)$ & $11.9(3.8)$ \\
\hline
\end{tabular}

AUDIT: Alcohol Use Disorders Identification Test.

same assessment as MI patients (10-15 minutes) and were mailed a new feedback sheet identical to the one used in the MI booster.

\section{Data analysis}

Patients who were randomized to condition were followed-up and included in analyses, regardless of whether boosters were completed. Frequency distributions were evaluated for normality and outliers adjusted [31]. The driving after drinking variable was logtransformed to correct for positive skew. The alcoholrelated injuries measure was also positively skewed but remained skewed after transformation, so was dichotomized. For continuous measures, repeated-measures analysis of variance (ANOVA) or analysis of covariance (ANCOVA) were conducted. Repeated-measures $\chi^{2}$ analysis was used for dichotomous outcomes.

Clinical significance was evaluated using the Jacobsen \& Truax [32] reliable change index which determines the reliability of change that occurred following an intervention by comparing change in participants from baseline to follow-up to the standard error of measurement of the outcome. The reliable change index $=x_{1}-x_{2} / \sqrt{ } 2\left(s_{1} \sqrt{ } 1-\right.$ $\left.\mathrm{r}_{\mathrm{xx}}\right)^{2}$, where $\mathrm{x}_{1}=$ baseline score, $\mathrm{x}^{2}=12$-month score, $\mathrm{s}_{1}=$ the standard deviation of the baseline score and $\mathrm{r}_{\mathrm{xx}}=$ the test-retest reliability of the measure (0.96) [33]. Jacobsen \& Truax also recommend defining a cut-off point to establish when an individual's behavior falls into a normal distribution. In our sample, using a cut-off 2 standard deviations (SD) from the baseline mean of average drinks per week (recommended by Jacobsen \& Truax when a normative population is not available) resulted in a cut-off of 0 . As this is not a reasonable cut-off we did not use a cut-off score, which limited us only in that we were not able to establish the proportion of individuals who were 'recovered' (i.e. reliably below the cutoff), a distinction not particularly important in this sample. Using a 95\% confidence interval around the baseline score, we determined the proportion of participants in each condition who were reliably improved or reliably worse.

\section{RESULTS}

A total of 198 patients (111 BAC positive, 40 self-report positive and 47 AUDIT-positive) were randomized. Infor- mation about these three groups is presented in Table 2 . Patient eligibility and enrollment are shown in Fig. 1.

\section{Baseline differences}

Patients' descriptive information is presented in Table 3. Patients in FO had more years of school, but no other significant differences in demographics, consumption or alcohol problems between the conditions were detected. There were no significant differences in the proportion of patients assigned to the intervention conditions for each counselor.

\section{Treatment fidelity}

Items reflecting non-specific counseling techniques and session quality (i.e. 'the counselor was easy to talk to', 'the counselor gave me the chance to ask questions' and 'how satisfied were you with the session?') did not differ between conditions but MI patients, compared to FO, gave higher ratings for the counselors on MI-consistent topics (Table 1).

\section{Booster sessions}

Booster 1 was completed by $81.6 \%$ of patients in $\mathrm{MI}$ and $92.0 \%$ in FO, $\chi_{(1, n=198)}^{2}=4.66, P<0.05$. Booster 2 was completed by $73.5 \%$ of patients in $\mathrm{MI}$ and $90.0 \%$ in $\mathrm{FO}$, $\chi^{2}(1, n=198)=9.09, P<0.01$.

\section{Follow-up assessments}

$\chi^{2}$ and $t$-test analyses showed that completion rates for 6and 12-month follow-up assessments $(83.3 \%$ and $81.3 \%$, respectively) did not differ by gender or condition. Six- and 12-month completers had higher baseline numbers of drinking days, $t_{(196)}=-2.48, P<0.05$, heavy drinking days, $t_{(196)}=-3.26, P<0.05$ and drinks per week, $t_{(196)}=-3.08, P<0.01$ than non-completers.

\section{Intervention outcomes}

Separate repeated-measures ANOVAs were conducted on number of days drinking, heavy drinking days and standardized drinks per week for the two follow-ups. For all these measures time effects were significant, with both groups significantly reducing their consumption from baseline to 6- and 12-month follow-up (see Table 4). In 


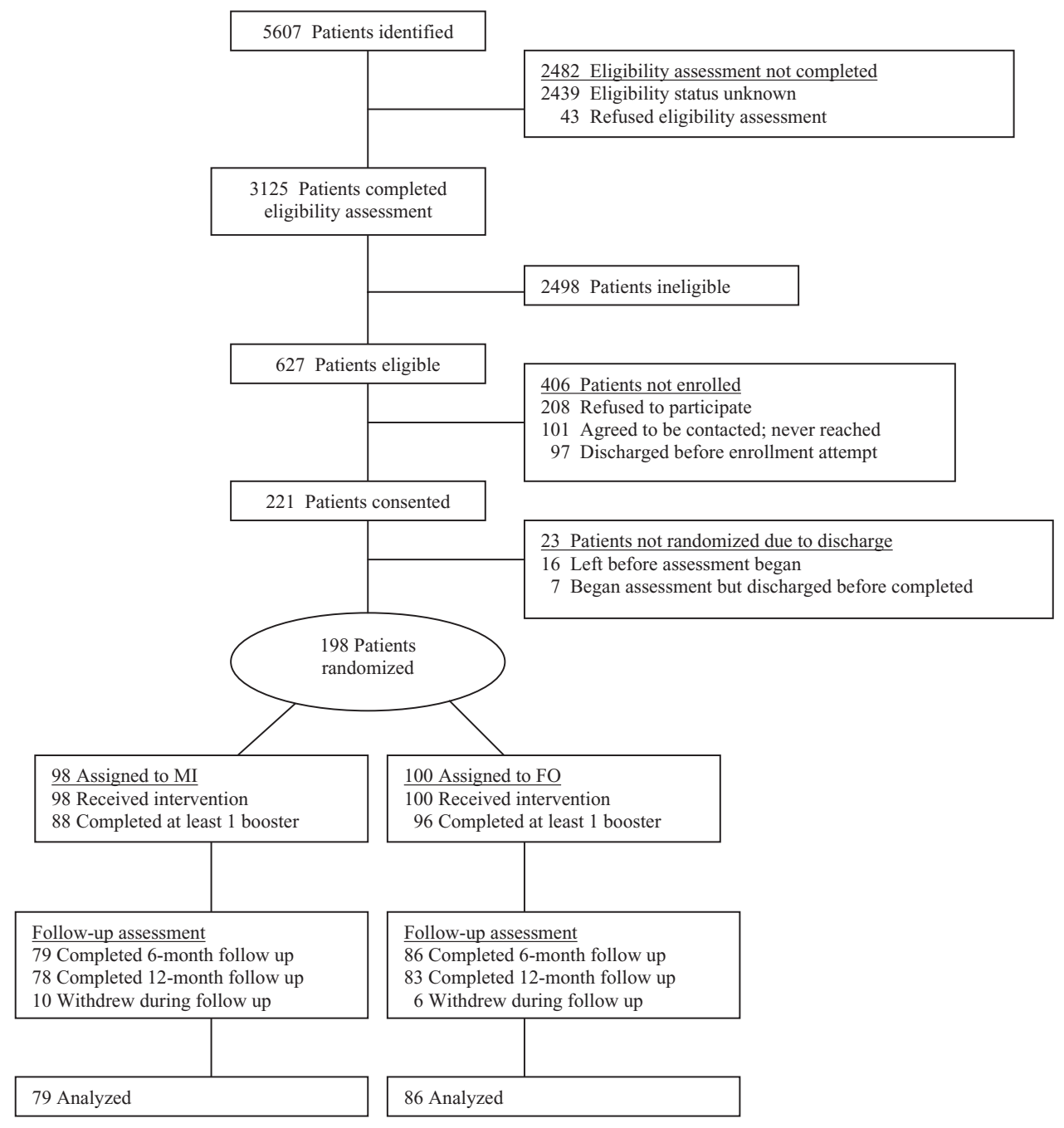

Figure I Flowchart of patient eligibility, enrollment and participation

addition, treatment $\times$ time interactions were found on all three consumption measures. Follow-up tests showed that the MI group had significantly greater reductions in consumption than FO on all three consumption measures at both 6 and 12 months (Table 4). Analyses of covariance were also conducted on the three consumption outcomes at 6 and 12 months, with the baseline value as the covariate, with similar results.

From baseline to 12 months MI patients reduced consumption by $45-53 \%$, depending on the measure, whereas FO reduced consumption by $11-18 \%$. The clinical significance evaluation of these findings using the Jacobsen \& Truax [32] method indicated that 38.5\% of the patients in MI and $19.3 \%$ in FO had reliably reduced their use (drinks per week) at the 12-month follow-up, whereas $5.1 \%$ and $8.4 \%$ had reliably increased their use in MI and FO, respectively. Follow-up one-way ANOVAs on the three consumption outcomes at both follow-ups indicated that there were no counselor differences in outcomes.
An ANCOVA was conducted with 12-month RAPI scores using the baseline score as a covariate; treatment condition effects were not significant. Repeated-measures ANOVA was used to analyze the drinking and driving scores, with no significant results. Repeated-measures ANOVA on the number of moving violations revealed a significant time effect, $F_{(1,123)}=5.38, P<0.05$, indicating a reduction in moving violations at the 12-month followup but no time $\times$ condition interaction. Alcohol-related driving violations were very low $(3.7 \%$ of the sample at baseline and $1.6 \%$ at follow-up), so were not analyzed between groups. A repeated-measures $\chi^{2}$ analysis with the alcohol-related injury data revealed a significant time effect, $\chi_{(1, n=138)}^{2}=36.75, P<0.001$, reflecting a reduction in alcohol-related injuries across conditions with no condition $\times$ time interaction. A repeated-measures $\chi^{2}$ analysis was also conducted on additional treatment which revealed a significant time effect, $\chi_{(1, n=155)}^{2}=6.55$, $P<0.05$, reflecting greater treatment-seeking following intervention, but no time $\times$ condition interaction. 
Table 3 Description of participants.

\begin{tabular}{|c|c|c|c|c|c|}
\hline Measure & $\begin{array}{l}M I(n=98) \\
M(S D) \text { or percentage }\end{array}$ & $\begin{array}{l}F O(n=100) \\
M(S D) \text { or percentage }\end{array}$ & $d f$ & $t$ & $\chi^{2}$ \\
\hline Male & $68(69.4 \%)$ & $66(66.0 \%)$ & 1 & & 0.26 \\
\hline Age & $20.7(1.8)$ & $20.4(1.9)$ & 196 & 0.99 & \\
\hline \multicolumn{6}{|l|}{ Race } \\
\hline White & $60(61.2 \%)$ & $70(70.0 \%)$ & 5 & & 6.31 \\
\hline Hispanic & $13(13.3 \%)$ & $12(12.0 \%)$ & & & \\
\hline Black & $5(5.1 \%)$ & $8(8.0 \%)$ & & & \\
\hline Asian & $2(2.0 \%)$ & $1(1.0 \%)$ & & & \\
\hline American Indian & $3(3.1 \%)$ & $0(0.0 \%)$ & & & \\
\hline Other or multiple race & $15(15.3 \%)$ & $9(9.0 \%)$ & & & \\
\hline Years of education & 11.9 & $12.7(1.7)$ & 196 & $3.07^{* *}$ & \\
\hline $\mathrm{BAC}$ in ED & $0.083(0.085)$ & $0.081(0.086)$ & 196 & 0.23 & \\
\hline \multicolumn{6}{|l|}{ Reason for treatment } \\
\hline Assault/fight & $23(23.7 \%)$ & $26(26.0 \%)$ & 5 & & 0.94 \\
\hline Motor vehicle crash & $21(21.6 \%)$ & $21(21.0 \%)$ & & & \\
\hline Fall & $11(11.3 \%)$ & $12(12.0 \%)$ & & & \\
\hline Other injury & $19(19.6 \%)$ & $23(23.0 \%)$ & & & \\
\hline Illness & $3(3.1 \%)$ & $2(2.0 \%)$ & & & \\
\hline Substance use only & $20(20.6 \%)$ & $16(16.0 \%)$ & & & \\
\hline
\end{tabular}

${ }^{* *} P<0.01$. MI: motivational interviewing; FO: feedback only; BAC: blood alcohol concentration; ED: emergency departments.

\section{Collateral reports}

Collateral reports were completed for $126(76.4 \%)$ and $125(77.6 \%)$ of patients who completed 6- and 12-month follow-ups, respectively. Correlation between patient and collateral report on number of heavy drinking days in the past 6 months was $0.54(P<0.001)$ at 6 months and $0.59(P<0.001)$ at 12 -month follow-up. Correlation between patient and informant on alcohol problems (RAPI score) was $0.48(P<0.001)$ at 6 months and $0.44(P<0.001)$ at 12 months. Between-groups analyses conducted on the collateral report on number of heavy drinking days, covarying the participant's report of heavy drinking days at baseline, resulted in a significant between-groups difference at 6 months, $F_{(1,102)}=4.94$, $P<0.05$, but not quite a significant difference at 12 months, $F_{(1,109)}=3.60, P<0.06$, both favoring MI.

\section{DISCUSSION}

Reductions in alcohol use, alcohol-related injuries and moving violations, and increases in treatment seeking, were seen in alcohol-involved young adults following our brief interventions in a busy ED. Administering a full MI showed greater efficacy than FO on several measures of consumption, an effect that persisted up to 12 months. The magnitude of the effect of MI was striking: patients who received the full MI reduced their consumption three to four times as much as FO patients, and over one-third of the MI group showed clinically significant and reliable improvement in consumption 1 year following the inter- vention. However, there were non-significant differences between groups at baseline on some variables which may have given participants in the MI group more 'room' to reduce their drinking. Therefore, interpretation of the within-groups effects on these variables should be made with caution. Nevertheless, FO does not appear to improve outcomes in our sample of young adults. Enrollment of participants was inclusive of patients who either had alcohol detected in the ED or a history of alcohol problems. Thus, the heterogeneity of this sample supports the external validity as well as the generalizability of our findings.

That we did not find group differences on alcohol problems is somewhat puzzling, especially as an earlier study of MI with a similar age group showed an impact on alcohol-related negative consequences, but not consumption, after 6 months [16], although this inconsistency in findings across studies is not unusual [34]. It is possible that the personalized feedback had a direct harm reduction effect, whereas the in-person MI was necessary to affect alcohol use. Alternatively, effects on use may have emerged as a result of the boosters. There is evidence that alcohol use is reduced among patients for several months following discharge from an ED [35], suggesting that a one-session MI may add relatively little to the motivational effects of ED treatment [15]. Boosters may have strengthened the effect of the intervention and allowed effects on use to emerge, providing support for clinical guidelines that recommend telephone or in-person follow-up after MI. 


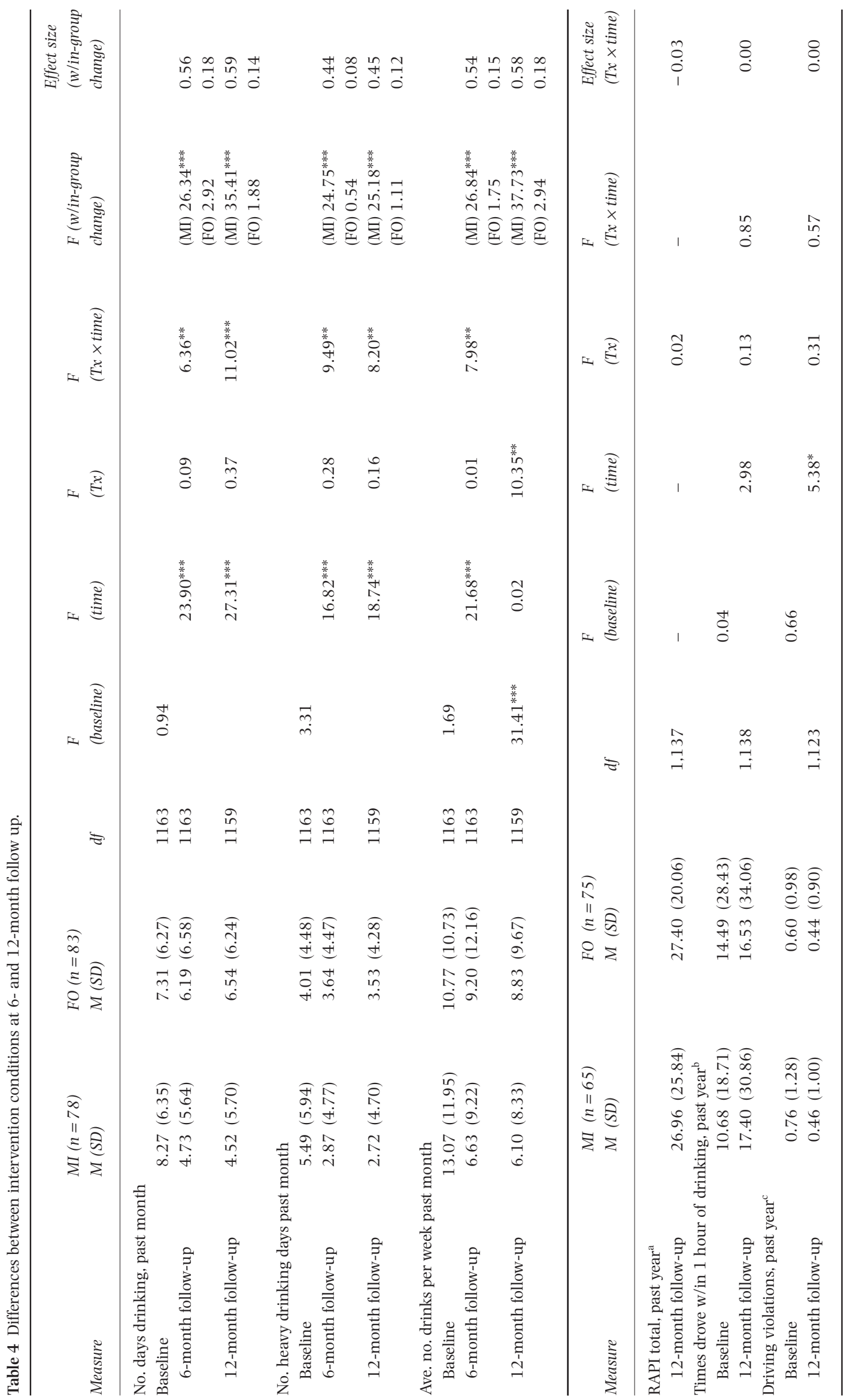




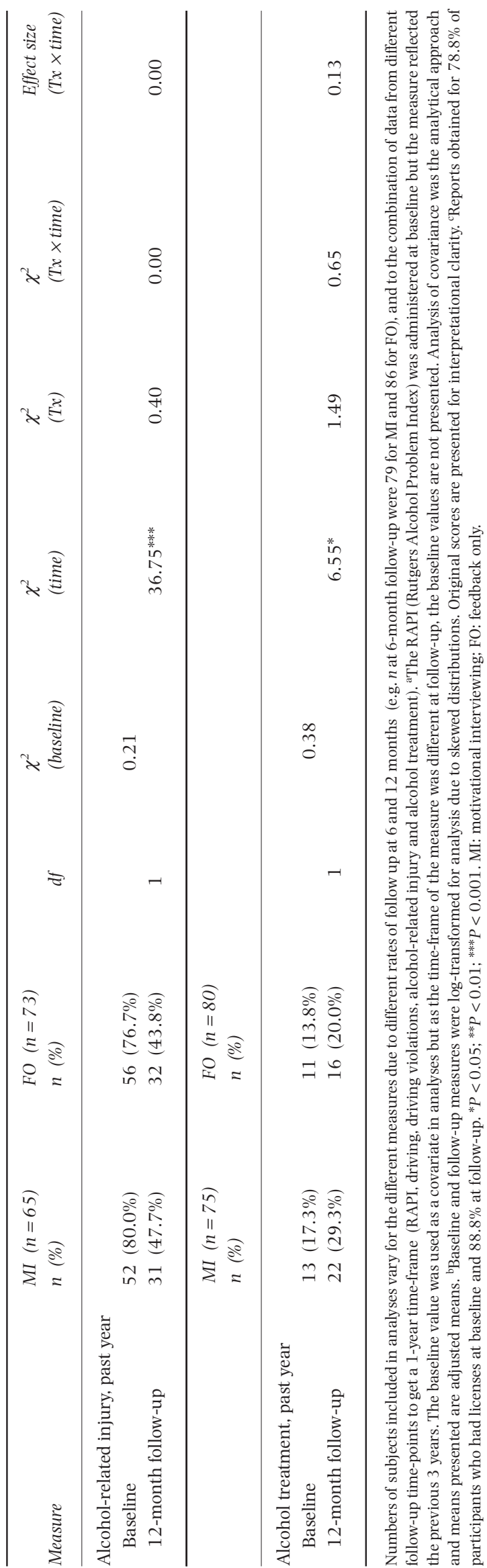

Alternatively, the time effects that we observed could be a reaction to the trauma of the event, to the overall treatment received in the $\mathrm{ED}$, to the assessment procedures, or to a combination of these factors. Reactivity to assessment procedures is certainly a possible explanation, as has been illustrated recently in MI findings with a college student population [36].

Further elaboration on the study's limitations is warranted. It is important to note that we did not have a no-treatment control group in this study and, as mentioned above, nor could we control for the effects of our assessment battery or medical treatment in the ED itself. Both these factors may lead to reductions in alcohol use and related behavior. Further, the lack of a control group prevents us from being able to conclude that the reductions in consumption that occurred in both groups were a function of our interventions. The lack of equivalence in counselor contact time between conditions also allows for an alternative explanation of our favorable findings for MI. Nevertheless, given the evidence in support of MI with this population and the mandate to conduct brief interventions with alcohol-involved patients treated in trauma settings, we believe including a no-treatment group or controlling for contact time were less important than testing a briefer ecologically valid approach in this applied setting.

It is also important to note that it would have been ideal to have objective verification of fidelity, but audiotaping treatment was not feasible in our ED. Indeed, we started the study audiotaping sessions but found that the tapes could not be rated confidently due to the quality of the recordings, so we discontinued audiotaping. Nevertheless, patient assessments of treatment content and process suggested that MI principles were being followed. An additional limitation of the present study is that the sample was limited to young adults, and the results may not generalize to other age groups.

Another concern is that the FO group completed boosters at higher rates than did the MI group. However, if the boosters reduce risk for future alcohol consumption, this difference in completion rates should serve to make the groups more similar and therefore our results may actually underestimate the effects of the MI. Finally, this study did not address experimentally the effect of adding the boosters to the MI, so we are unable to state whether the additional contact had incremental effect over the baseline session.

In sum, this study is the first to compare two active interventions with ED patients and to demonstrate an effect on alcohol use among young adults in an ED. While both the interventions studied hold promise as lowintensity methods for reducing alcohol-related injuries and mortality in this high-risk population, only the full MI impacted alcohol consumption through a 12-month 
follow-up. Nevertheless, it is possible that FO is the more cost-effective option. Unfortunately, limitations in our methodology preclude our being able to test this hypothesis. Future studies should replicate these results and determine the cost-effectiveness of a full MI compared to feedback only when delivered in an ED setting.

\section{Acknowledgements}

This investigation was supported by research grant AA09892 from the National Institute on Alcohol Abuse and Alcoholism and by a Department of Veterans Affairs Senior Career Research Scientist Award to Peter M. Monti. Portions of this study were presented at the 35th Annual Meeting of the Western Trauma Association, Jackson Hole, WY, March 2005.

\section{References}

1. Substance Abuse and Mental Health Services Administration (SAMHSA). Results from the National Survey on Drug Use and Health: National Findings. Office of Applied Studies, NSDUH Series H-25, DHHS Publication no. SMA 04-3964. Rockville, MD; SAMHSA; 2004.

2. Hingson R. W., Heeren T., Winter M., Wechsler H. Magnitude of alcohol-related morbidity and mortality among U.S. college age students 18-24: changes from 1998 to 2001. Апnи Rev Public Health 2003; 26: 259-79.

3. Substance Abuse and Mental Health Services Administration (SAMHSA). Results from the National Survey on Drug Use and Health: National Findings. Office of Applied Studies, NSDUH Series H-28, DHHS Publication no. SMA 05-4062. Rockville, MD; SAMHSA; 2005.

4. Rivara F. P., Jurkovich G. J., Gurney J. G., Seguin D., Fligner C. L., Ries R. et al. The magnitude of acute and chronic alcohol abuse in trauma patients. Arch Surg 1993; 128: 907-13.

5. Soderstrom C. A., Smith G. S., Dischinger P. C., McDuff D. R., Hebel J. R., Gorelick D. A. et al. Psychoactive substance use disorders among seriously injured trauma center patients. JAMA 1997; 277: 1769-74.

6. D'Onofrio G., Degutis L. C. Screening and brief intervention for alcohol problems in the emergency department: a systematic review. Acad Emerg Med 2002; 9: 627-38.

7. Gentilello L. M., Rivara F. P., Donovan D. M., Jurkovich G. J., Daranciang E., Dunn C. W. et al. Alcohol interventions in a trauma center as a means of reducing the risk of injury recurrence. Ann Surg 1999; 230: 473-80.

8. Babor T. F., Higgins-Biddle J. C. Alcohol screening and brief intervention: dissemination strategies for medical practice and public health. Addiction 2000; 95: 677-86.

9. National Center for Injury Prevention (NCIP) and Control Centers for Disease Control and Prevention (CCDCP). Recommendations for alcohol screening and intervention in the emergency department. In: Hungerford D. W., Pollock D. A., editors. Alcohol problems among emergency department patients. Atlanta: NCIP/CCDCP; 2002, p. 9-13.

10. American College of Surgeons. Resources for Optimal Care of the Injured Patient. Chicago, IL: American College of Surgeons Press, in press.

11. Miller W. R., Rollnick S. Motivational Interviewing: Preparing
People to Change Addictive Behavior, 2nd edn. New York: Guilford Press; 2002.

12. Miller W. R., Wilbourne P. L., Hettema J. E. What Works? a Summary of Alcohol Treatment Outcome Research. In: Hester R. K., Miller W. R., editors. Handbook of alcoholism treatment approaches, 3rd edn. Boston, MA: Allyn \& Bacon; 2003, p. 13-63.

13. Vasilaki E. I., Hosier S. G., Cox W. M. The efficacy of motivational interviewing as a brief intervention for excessive drinking: a meta-analytic review. Alcohol Alcohol 2006; 41: 328-35.

14. Larimer M. E., Cronce J. M. Identification, prevention and treatment: a review of individual-focused strategies to reduce problematic alcohol consumption by college students. J Stud Alcohol Suppl 2002; 14: 148-63.

15. Longabaugh R., Woolard R., Nirenberg T., Minugh P. A., Becker B., Clifford P. et al. Evaluating the effects of a brief motivational intervention for injured drinkers in the emergency department. J Stud Alcohol 2001; 62: 806-16.

16. Monti P. M., Colby S. M., Barnett N. P., Spirito A., Rohsenow D. J., Myers M. et al. Brief intervention for harm reduction with alcohol-positive older adolescents in a hospital emergency department. J Consul Clin Psychol 1999; 67: 989-94.

17. Spirito A., Monti P., Barnett N. P., Colby S. M., Sindelar H., Rohsenow D. J. et al. A randomized clinical trial of a brief motivational intervention for alcohol positive adolescents. J Pediatr 2004; 145: 396-402.

18. Lewis M. A., Neighbors C. Social norms approaches using descriptive drinking norms education: a review of the research on personalized normative feedback. $J$ Am Coll Health 2006; 54: 213-8.

19. Miller W. R. Enhancing Motivation for Change in Substance Abuse Treatment. Treatment Improvement Protocol (TIP) Series, DHHS Publication no. 99-3354. 1999. Rockville, MD: DHHS

20. Agostinelli G., Brown J. M., Miller W. R. Effects of normative feedback on consumption among heavy drinking college students. J Drug Educ 1995; 25: 31-40.

21. Murphy J. G., Benson T. A., Vuchinich R. E., Deskins M. M., Eakin D., Flood A. M. et al. A comparison of personalized feedback for college student drinkers delivered with and without a motivational interview. J Stud Alcohol 2004; 65: 200-3.

22. Walters S. T., Neighbors C. Feedback interventions for college alcohol misuse: what, why, and for whom? Addict Behav 2005; 30: 1168-82.

23. Miller W. R., Westerberg V. S., Waldron H. B. Evaluating alcohol problems in adults and adolescents. In: Hester R. K., Miller W. R., editors. Handbook of alcoholism treatment approaches, 2nd edn. Boston, MA: Allyn \& Bacon; 1995, p. 61-88.

24. US Department of Health and Human Services. Guidelines for Treatment of Alcohol and Other Drug-Abusing Adolescents. Center for Substance Abuse Treatment, Treatment Improvement Protocol Series, Publication no. 93-2010. Washington, DC: US Government Printing Office; 1993.

25. Saunders J. B., Aasland O. G., Babor T. F., De La Fuente J. R., Grant M. Development of the alcohol use disorders identification test (AUDIT): WHO collaborative project on early detection of persons with harmful alcohol consumptionII. Addiction 1993; 88: 791-804.

26. Sobell L. C., Sobell M. B. Alcohol Timeline Followback Users' Manual. Toronto, Canada: Addiction Research Foundation; 1995. 
27. White H. R., Labouvie E. W. Towards the assessment of adolescent problem drinking. J Stud Alcohol 1989; 50: 30-7.

28. Spirito A., Rasile D., Vinnick C., Jelalian E., Arrigan M. E. Relationship between substance abuse and self-reported injuries among adolescents. J Adolesc Health 1997; 21: 221-4.

29. Donovan J. E. Young adult drinking-driving: behavioral and psychosocial correlates. J Stud Alcohol 1993; 54: 600-13.

30. Miller W. R., Sovereign R. G., Krege B. Motivational interviewing with problem drinkers. II. The drinker's check-up as a preventive intervention. Behav Psychother 1988; 16: 25168.

31. Tabachnick B. G., Fidell L. S. Using Multivariate Statistics, 3rd edn. New York: Harper Collins; 1996.

32. Jacobson N. S., Truax P. Clinical significance: a statistical approach to defining meaningful change in psychotherapy research. J Consult Clin Psychol 1991; 59: 12-19.
33. Sobell L. C., Sobell M. B., Leo G. I., Cancilla A. Reliability of a timeline method: Assessing normal drinkers' reports of recent drinking and a comparative evaluation across several populations. Br J Addict 1988; 83: 393-402.

34. Dinh-Zarr T., Goss C., Heitman E., Roberts I., DiGuiseppi C. Interventions for preventing injuries in problem drinkers. Cochrane Database Syst Rev 2004; Issue 3: CD001857. DOI: 10.1002/14651858CD001857.pub2.

35. Dunn C., Zatzick D., Russo J., Rivara F., Roy-Byrne P., Ries R. et al. Hazardous drinking by trauma patients during the year after injury. J Trauma 2003; 54: 707-12.

36. Carey K. B., Carey M. P., Maisto S. A., Henson J. M. Brief motivational interventions for heavy college drinkers: a randomized controlled trial. J Consult Clin Psychol 2006; 74: 943-54. 